${ }^{2} \mathbf{K}$ E Rogstad, ${ }^{3} \mathrm{G}$ Hughes, ${ }^{4} \mathrm{G}$ Debelle, $5 \mathrm{~J}$ Evans, ${ }^{6} \mathrm{R}$ Reading. ${ }^{\text {SSheffield Teaching }}$ Hospitals NHS Foundation Trust, Sheffield, UK, '2University of Sheffield Medical School, Shefield, UK, ${ }^{3}$ STI Section, Health Protection Services Colindale, Health Protection Agency, London, UK, 'Birmingham Childrens' Hospital, Birmingham, UK, ${ }^{5}$ Department of Genitourinary Medicine, Norfolk and Norwich University Hospital, Norwich, UK, ${ }^{6}$ Jenny Lind Paediatric Dept, Norfolk and Norwich University Hospital, Norwich, UK

Background Children who have been sexually abused (CSA) are potentially at risk of sexually transmitted infections (STI). It is not known how frequently such infections are identified within the population nor whether the implications of the mode of transmission are recognised and investigated appropriately.

Methods Active surveillance occurred through the British Paediatric Surveillance Unit system (www.rcpch.ac.uk/bpsu) which covers all paediatricians (estimated $>95 \%$ ) in UK and Republic of Ireland. Consultant paediatricians were asked to report cases with laboratory confirmed Neisseria gonorrhoeae (Ng), Treponema pallidum (Tp), Chlamydia trachomatis (Ct) or Trichomonas vaginalis (Tv) in children aged 1 to 12 years between January 2010 and January 2012. Anyone reporting a case was sent a clinical questionnaire. The adequacy of the initial and confirmatory diagnostic tests was judged against relevant national guidelines. Child protection investigations undertaken were arranged into a hierarchical classification.

Results Fifteen cases were reported - $7 \mathrm{Ng}, 6 \mathrm{Ct}, 1 \mathrm{Tp}$ and $1 \mathrm{Tv}$. Fourteen presented because of symptoms ( 5 with ophthalmic symptoms), 3 had isolated ophthalmic infections), 1 following alleged CSA. Eleven of 15 had other indicators of possible CSA including allegation, behavioural or previous child protection concerns. Tests used were adequate and all had additional STI testing undertaken including $10 \mathrm{HIV}$ and $12 \mathrm{Tp}$ and hepatitis B. All but one case were referred for multi-agency child protection investigations, in three cases sexual CSA was confirmed at court or case conference (some outcomes awaited).

Conclusion This is the first population-based study of bacterial STI incidence in under 13 year-olds in the UK. Incidence was very low. Once detected, there are high levels of screening for other STIs using appropriate tests in line with national guidelines, and assessments for CSA. This is an improvement on a previous study on HSV1 and may be a result of better guidance and evidence base.

\section{P3.008 EPIDEMIOLOGICAL CORRELATES OF CHLAMYDIA PGP3 ANTIBODY IN A PROSPECTIVE COHORT OF MEN AND WOMEN}

doi:10.1136/sextrans-2013-051184.0468

\begin{abstract}
.2P Horner, ${ }^{3} \mathrm{~N}$ Dickson, ${ }^{4} \mathrm{G}$ Wills, ${ }^{3} \mathrm{~A}$ Righarts, ${ }^{4} \mathrm{~S}$ Vieira, ${ }^{3} \mathrm{~S}$ Young, ${ }^{4} \mathrm{M}$ McClure 'University of Bristol, Bristol, UK; ' University Hospitals Bristol NHS Foundation Trust, Brsitol, UK; ${ }^{3}$ University of Otago, Dunedin, New Zealand; ${ }^{4}$ Imperial College London, London, UK
\end{abstract}

Background Epidemiological correlates of chlamydia (CT) antibody were investigated in a longitudinal cohort of just under 1000 men and women born in Dunedin, New Zealand in 1972/1973 at ages 26, 32 and 38 .

Methods Subjects were questioned on sexual behaviour and sexually transmitted infections (STIs) at ages 21, 26, 32 and 38 (19932011), and sera collected at ages 26, 32 and 38 for CT antibody. All sera were assayed by Pgp3 ELISA, and the age 32 samples by MOMP peptide ELISA, and assayed blinded. Ethical approval was obtained. Results Pgp3 antibody was strongly associated with history of CT, but not other STIs ( $p>0.3$ ). This association was much stronger for women ( $p=<0.001$, OR 8, 95\% CI 4-16.1) than men ( $p=0.07$, OR $2.64,95 \%$ CI $0.82-8)$. At age $26,17.4 \%(72 / 411)$ of all the women were Pgp3 sero-positive, as were $56.8 \%(25 / 44)$ of those giving a history of CT infection. For both men and women at age 26, Pgp3 antibody correlates with age at first intercourse and the number of partners. More women who were seropositive at age 26 lost Pgp3 antibody between the ages of 26 and $32(25 / 67,37.3 \%)$, than did seropositive women between 32 and 38 (7/56,12.5\%) ( $p=0.003)$. At age 32 women with previous CT infection were more likely to have Pgp3 antibody $(23 / 52,44.2 \%)$ than MOMP antibody (12/52, 23.1\%).

Conclusion Pgp3 antibody in women is strongly associated with past diagnosed CT infection, and at age 32 a more sensitive measure than MOMP antibody. It is associated with earlier age of first sexual intercourse and increasing number of partners, but not with a past history of other STIs. Pgp3 antibody prevalence declined over time. These data provide further information to show that Pgp3 antibody provides a measure of past CT infection.

\section{P3.009 PREVALENCE OF CHLAMYDIA TRACHOMATIS AND NEISSERIA GONORRHOEA INFECTION IN PREGNANT WOMEN ENROLLED IN A LARGE MULTICENTER CLINICAL TRIAL}

doi:10.1136/sextrans-2013-051184.0469

J Osiecki, J Duncan, M Lewinski, 0 Liesenfeld. Roche Molecular Systems, Pleasanton, CA, United States

Background Pregnant women infected with sexually transmitted diseases are at higher risk for miscarriage, pre-term delivery, low birth weight, and morbidity in the neonate associated with transmission of pathogenic agents. Treatment guidelines recommend screening pregnant women for Chlamydia trachomatis (CT) and Neisseria gonorrhoea (NG) on the first prenatal visit. This study was performed to determine the frequency of CT and NG infection observed in pregnant women enrolled in a large clinical trial study population.

Methods This multicenter retrospective cohort analysis was performed with data collected during the VENUS clinical trial, a study characterising the clinical performance of the cobas ${ }^{\circledR}$ CT/NG Test on the cobas ${ }^{\circledR} 4800$ system. Two FDA-cleared nucleic acid amplification tests (NAATs) were used as comparator assays. Obstetrics-gynaecology practises, family planning clinics, and STD clinics from diverse settings in the United States served as specimen collection sites. Patient infection status (PIS) was defined as positive when results from NAATs with different target regions generated positive results with collected samples.

Results Of 5,269 enrolled participants, 281 of 4315 eligible women (6.5\%) were found to be positive for CT infection and 69 of 4314 (1.6\%) were positive for NG according to PIS outcomes. Alternatively, 16 of 178 eligible pregnant women $(9.0 \%)$ were positive for CT, where 2 of 178 pregnant women (1.1\%) were considered positive for NG by PIS.

Conclusion Screening of pregnant women for CT and NG with the cobas $^{\circledR}$ CT/NG Test and two additional NAATs during the VENUS clinical trial revealed the prevalence of CT and NG infections are comparable to rates observed in the general female population.

\section{P3.010 COMPARISON OF THE RATE OF HOSPITALISATION FOR PELVIC INFLAMMATORY DISEASE (PID) FOLLOWING A DIAGNOSIS OF CHLAMYDIA OR GONORRHOEA IN WOMEN RESIDENT IN NEW SOUTH WALES, AUSTRALIA}

doi:10.1136/sextrans-2013-051184.0470

'J Reekie, 'B Donovan, 'R Guy, ${ }^{2} \mathrm{~J}$ Hocking, ${ }^{3} \mathrm{~L}$ Jorm, ${ }^{1} \mathrm{~J}$ M Kaldor, ${ }^{1,4} \mathrm{~J}$ Ward, 'B Liu, on behalf of the chlamydia \& reproductive health outcome investigators. 'University of New South UK, Sydney, Australia; 'University of Melbourne, Melbourne, Australia; 'University of Western Sydney, Sydney, Australia; 'Baker Institute, Alice Springs, Australia

Aim Studies have demonstrated and quantified the relationship between chlamydia infection and pelvic inflammatory disease (PID). However there is relatively little information regarding the 
rate of PID following gonorrhoea infection. This analysis compares the rate of hospitalisation for PID following a diagnosis of gonorrhoea to the rate following a chlamydia diagnosis.

Method All women, aged 15-45 years, in New South Wales (NSW), with a diagnosis of chlamydia or gonorrhoea between $1 / 7 / 2000$ and $31 / 12 / 2008$ were followed for up to one year after diagnosis for hospitalisations for PID. The incidence rates of PID hospitalisation among women with a chlamydia or gonorrhoea diagnosis were compared to the whole of the NSW population using standardised incidence ratios (SIRs). Poisson regression was used to compare the rates of PID hospitalisation after adjusting for age, diagnosis date, socioeconomic group, area of residence and prior births.

Results There were 38379 women with a chlamydia diagnosis. During 35014 person years of follow-up (PYFU), 485 were hospitalised for PID; incidence rate (IR) 13.8 per 1000 PYFU (95\% CI 12.615.1). Among 1023 women with a gonorrhoea diagnosis, during 895 PYFU 45 were hospitalised for PID; (IR 50.3 per 1000 PYFU, 95\% CI 35.6-65.0). Compared to the age-equivalent NSW female population, the incidence of PID hospitalisation was 27.0 (95\% CI 24.429.8) times greater among women who had a chlamydia diagnosis in the year prior to hospitalisation and 95.8 (95\% CI 64.2-137.6) times greater among women with a gonorrhoea diagnosis. Younger age, diagnosis prior to 2005, socioeconomic disadvantage and prior births were also associated with an increased rate of PID hospitalisation.

Conclusion Hospitalisation rates for PID were over 3 times greater in women diagnosed with gonorrhoea than chlamydia, and rates in both were substantially higher than in the general female population. Our results suggest that gonorrhoea causes more serious reproductive health sequelae than chlamydia.

\section{P3.011 DRY SWAB EVALUATION BY ROCHE 4800 CT/NG AND THE PRESTO-PLUS: CROSS-SECTIONAL STUDY OF GENITAL, RECTAL AND PHARYNGEAL CHLAMYDIA AND GONORRHOEA INFECTION IN WOMEN IN RURAL SOUTH AFRICA}

doi:10.1136/sextrans-2013-051184.0471

'D J de Waaij, ${ }^{1,2}$ J Dubbink, ${ }^{1,2} \mathrm{~L}$ van der Eem, ' $\mathrm{M}$ L A Bos, 'S Ouburg, ${ }^{3,4} \mathrm{D}$ A Lewis, ${ }^{2} \mathrm{H}$ Struthers, $2,5 \mathrm{~J}$ A McIntyre, 1,6S A Morré, ${ }^{2} \mathrm{R}$ P H Peters. ' $\mathrm{VU}$ University Medical Center, Amsterdam, The Netherlands; ${ }^{2}$ Anova Health Institute, Johannesburg and Tzaneen, South Africa; ${ }^{3}$ National Institute for Communicable Diseases, National Health Laboratory Service, Johannesburg, South Africa; ${ }^{4}$ University of the Witwatersrand, Johannesburg, South Africa; ${ }^{5}$ University of Cape Town, Cape Town, South Africa; ${ }^{6}$ University of Maastricht, Maastricht, The Netherlands

Backgound Epidemiological data, required to inform the design and implementation of control programmes, in relation to genital Chlamydia and Gonorrhoea in rural Africa, are limited. There are no data on the prevalence of rectal or pharyngeal infections among women. We evaluate the use of dry swabs by Roche 4800 CT/NG and the PRESTO-PLUS to determine the prevalence in three anatomic locations of chlamydial and gonococcal infections in African women.

Methods Cross-sectional study of 604 women visiting 25 randomly selected primary healthcare (PHC) facilities. Participants were recruited between November 2011 and February 2012. Setting: PHC facilities across rural Mopani District in Limpopo Province, South Africa. Participants: Women aged 18-49 years who reported at least one sex act in the past 6 months were eligible. A questionnaire was administered and physical examination conducted. Vaginal, anorectal and oropharyngeal swabs were tested for Chlamydia trachomatis and Neisseria gonorrhoeae.

Results 480 (including 5 times 4 controls) samples have been tested by both assays at this moment, the others are in progress. Overall prevalence of either infection was $20 \%$. Prevalence of genital chlamydia was $13 \%$ and gonorrhoea $5 \%$; rectal chlamydial infection was diagnosed in $4 \%$ and gonococcal in $2 \%$ of women. Clear geographical differences were observed in the CT and NG prevalances. Roche and PRESTO-PLUS had similar prevalences with slightly higher prevalances found by PRESTO-PLUS, however not all 604 samples have been tested, and discrepancy analyses will be performed in the upcoming 2-3 months.

Conclusion Dry swab collection seems a reliable method of sampling without majot prevalence differences between Roche and PRESTO-PLUS. Genital and rectal, but not pharyngeal, chlamydia and gonorrhoea infections are highly prevalent and frequently asymptomatic in women in rural South Africa. Young women attending healthcare facilities for antenatal care or family planning should be prioritised in control efforts.

\section{P3.012 HOW ROBUST ARE THE DESCRIPTIONS OF CHLAMYDIA NATURAL HISTORY USED IN ECONOMIC EVALUATIONS OF CONTROL STRATEGIES?}

doi:10.1136/sextrans-2013-051184.0472

'B Davies, 'S Anderson, ${ }^{2} \mathrm{~K}$ M E Turner, 'H Ward. 'Imperial College London, London, UK; ${ }^{2}$ University of Bristol, Bristol, UK

Background The decision to implement a Chlamydia screening programme is based on a detailed assessment of its projected impact and cost-effectiveness. In the absence of evidence from randomised controlled trials, transmission dynamic models are crucial to this process. However these models are highly sensitive to the representation of the infection. We review the evidence used to inform the model parameters highlighting their strengths and limitations.

Methods Published economic analyses of chlamydia screening interventions were identified following a systematic search of the literature. Only transmission dynamic models were included as they represent the gold standard. Parameters describing chlamydia infection were extracted and the variability across the studies assessed. The data used to inform each parameter was sourced and critically evaluated.

Results Eleven studies were included in this review, all evaluating chlamydia screening programme designs in developed countries. Many key natural history parameters are based on sparse historical data and there is wide variation in the values used across the models. For example,

- The per act transmission probability ranging from $3.75 \%$ to $15.3 \%$.

- The modelled duration of asymptomatic infection was between 180-370 days in women and 40-200 days in men.

- Only one paper includes a period of protective immunity following infection.

- Only 2 studies consider the role of reinfection in the development of complications

- However, there is a general consensus in the proportion of people that are asymptomatic; between $70-75 \%$ of women and $25-50 \%$ of men.

Conclusion We highlight the variability in descriptions of the natural history and emphasise the importance of using contemporary data to inform modelling studies. A clear consensus on the appropriate representation of the natural history is needed, with estimates continuously updated using new evidence.

\section{P3.013 THE ROLE OF CHLAMYDIA IN PELVIC INFLAMMATORY DISEASE AND ECTOPIC PREGNANCY}

doi:10.1136/sextrans-2013-051184.0473

${ }^{1} \mathbf{M}$ Price, ${ }^{2} \mathrm{~A}$ Ades, ${ }^{2} \mathrm{~N}$ Welton, ${ }^{3} \mathrm{~K}$ Soldan, ${ }^{4} \mathrm{P}$ Horner. ${ }^{1}$ University of Birmingham, Birmingham, UK; '2University of Bristol, Bristol, UK; ${ }^{3}$ Health Protection Agency, London, UK; ${ }^{4}$ University of Brisol, Bristol, UK 